$\mathrm{DOE} / \mathrm{CE} / 90029--7$

DE92 011607

\title{
EXPERIMENTAL STUDIES OF FRICTION AND HEAT TRANSFER IN ENHANCED PASSAGES
}

\author{
Progress Report \\ for Period October 1, 1991 - March 31, 1992
}

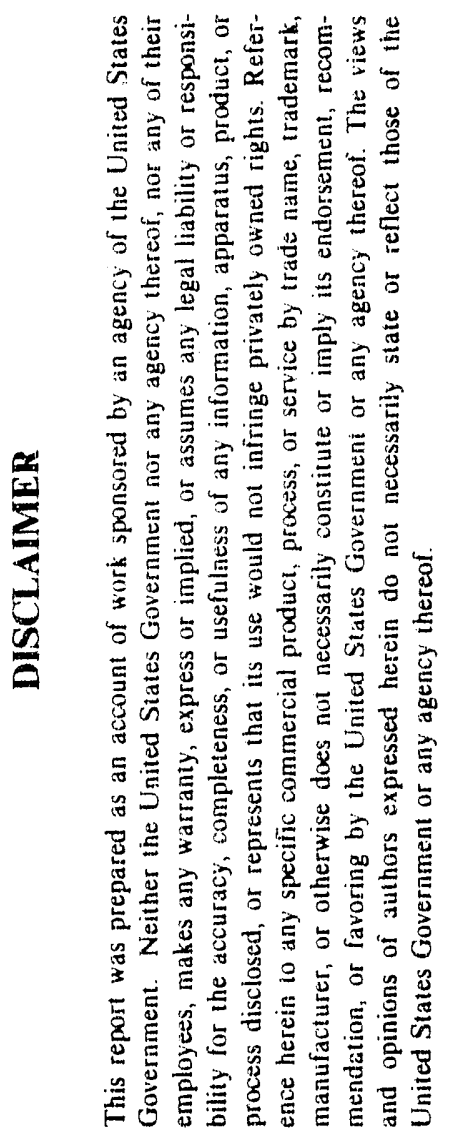

N. T. Obot, E. B. Esen and L. Das

March 1992

Prepared for

THE U. S. DEPARTMENT OF ENERGY

AGREEMENT NO. DE-FG02-89CE90029.A000

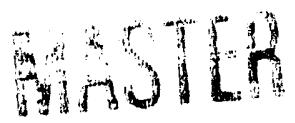




\section{Experimental Studies of Friction and Heat Transfer in Enhanced Passages}

\section{Introduction}

The objectives of this research are two-fold; one is to carry out an extensive experimental investigation of pressure drop and heat transfer for internally enhanced passages in laminar. transitional and turbulent flow. The laminar and transition regions bave received very meager treatment in the past. In addition to the effects of Reynolds number and the geometric characteristics of the enhanced surfaces, a comprehensive investigation of the effect of tluid properties. which is usually expressed in terms of the Prandtl number, is one of the central features of this research program. The influence of Prandtl number on friction and heat transfer is undoubtedly the least understood because only a handful of studies considered more than one fluid. Also, there has been no consistent investigation of the Prandtl number effect for lamirar or transitiorial flow.

The second objective is to develop a general method that will aid data correlation and prediction which, in turn, will provide better understanding friction and heat transfer characteristics for smooth and enhanced passages. More specifically, the aim is to validate the frictional law of corresponding states (DOE/CE/90029-2) using the present experimental data.

As noted in the last progress report ( DOE/CE/90029-6), a paper based on the spirally fluted tube results was presented at the 1991 National Heat Transfer Conference in Minneapolis. A modined version of this paper was published in the recent issue of a Int. Comm. Heat Mass Transfer, Vol. 19, \# 1, p. 41, 1992. Also, a paper on the smooth tube friction and heat transfer in laminar and transitional flow was submitted for possible publication in the above journal. And, further, a general paper on the frictional law of corresponding states was accepted for publication in Trans. IChemE, Chem. Eng. Res. 6i Design. Copies of these papers are appended to this report (Appendix 1).

It should perhaps also be mentioned that the P.I. devoted a considerable amount of time to support the Thermal Sciences Research at Argonne. Specifically, the corresponding states method was applied with remarkable success to two-phase flow frictional pressure drop data. Several papers resulting from these efforts are also appended to this report. (Appendix 2).

\section{Recent Progress}

As projected in the last progress report (DOE/CE/90029-6). air tests with the seven remaining spirally indented tubes (supplied by Yorkshire Alloys, England) were completed in December 1991. This brought the total number of tubes tested with air to 24, and marked the end of the air experiments. This phase of the research program represents the most extensive 
and consistent investigation of pressure drop and heat transfer ever undertaken for laminar. transitional and turbulent flow through internally enhanced passages.

Data analysis aimed at generalization of the air data is in progress, as is the preparation of the report on this aspect of the research program. One of the most important conclusions established from the analysis is that heat transfer coefficients for enhanced passages can be computed from the known values of the critical friction factor and Reynolds number at the onset of transition to turbulent flow, with errors that are, for the most part, under $\pm 10 \%$. The same conclusion can be inferred from the smooth tube results that are given in Appendix 1 (Paper \# 2).

The construction of the liquid test facility, shown schematically in Figure 1, and the calibrations of the two rotameters were completed. Qualification tests were made to establish the reliability of the instrumentation for laminar, transitional and turbulent flow pressure drop. The tests involved measurements of pressure drop with the two spirally fluted tubes also tested by Ravigururajan and Bergles (1986) with water.

Comparisons of our air and water data are given in Figures 2 and 3. In each figure, the water results of Ravigururajan and Bergles for the same tube are also shown, as are our smooth tube air data. Clearly, there are no significant differences between the enhanced tube data for air and water. Note that. Figure 2 shows water data for two replicate trials made on different days; the reproducibility of the results is good over the entire Reynolds number range. The results of Ravigururajan and Bergles for the same tubes lie well below those of the present study:

The documentation of the geometric characteristics of the various enhanced tubes were carried out as planned. This task was arcomplished in consultation with Dr. T. J. Rabas of Argonne National Laboratory. A summary of the geometric details of all 24 flow passages are given in Table 1, while Table 2 gives a summary of the test conditions for the air studies In the first column of Table 1 or 2, S, GA, HC, W, and Y denote smooth, General Atomic, Hitachi Cable, Wieland-Werke AG, and Yorkshire Alloys, respectively. The last four are the suppliers of the passages used in this study. Note that the test conditions for liquid are the same as those in Table 2 .

In summary, the highlights of the project status during the period covered by this report include:

- completion of the air tests with the seven remaining enhanced passages, bringing the total number tested to twenty-four.

- preparation and submission of four manuscripts. 
- the geometric characteristics of the enhanced passages have been documented in detail.

- construction of the liquid test facility and calibrations of the flowmeters.

- pressure drop measurements with two (General Atomic) spirally fluted tubes. Note that air heat transfer tests were not made with these tubes and such measurements will not be made with liquids.

- analysis of the air data and the preparation of the final report, both of which will be completed in the very near future.

Finally, there were problems with laminar flow pressure drop measurements with water. Unlike the air test facility that was less susceptible to vit ration, several heavy devices are piped into the liquid flow loop and the pipe sizes range from $57.8 \mathrm{~mm}$ to $19.1 \mathrm{~mm}$. Firm support of the structure did not eliminate the occurrence of unsteady prossure drop readings in laminar flow due to vibration and extraneous noise pick-up. The problem was resolved using amplifiers and active filters to control the ripple of the pressure transducer output.

\section{Quarterly Goals (March 1 - May 31, 1992)}

Two major goals are set for this period:

- The generalization of the air data and the preparation of the final report will be completed during this period. The data analysis is more involved than anticipated. For instance. it became evident from the analysis of the smooth tube data that the fricuion factor should be computed using the air density based on the average surface temperature instead of the usual practice of using the bulk mean temperature. This necessitated the re-evaluation of the friction factor as well as the relevant heat transfer data for for all flow passages.

- The goal is to complete qualification beat transfer tests with the smooth tube and to begin full-scale testing with the enhanced passages. During the academic year graduate research assistants (especially new graduate students) spend only a small fraction of their time on research because they must take courses. For this reason, the research assistant (Ms. L. Das), who joined this group in September 1991, is just beginning her research.

\section{Reference}

Ravigururajan, T. S. and Bergles, A. E., "Study of Water-Side Enhancement for Ocean Thermal Energy Conversion Heat Exchangers," HTL-44/ERI Project 1718. Iowa State University (1986). 


\begin{tabular}{|c|c|c|c|c|c|c|c|c|c|c|c|c|c|c|c|c|c|c|c|c|c|c|c|}
\hline 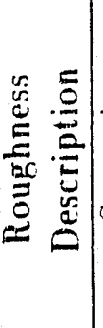 & & 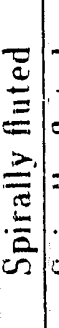 & 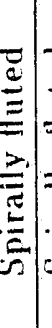 & 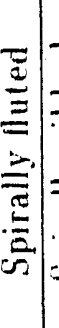 & 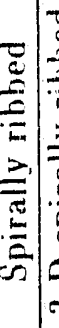 & 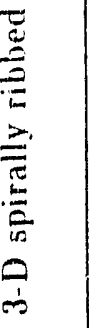 & 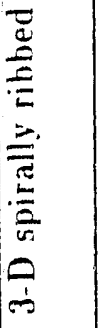 & 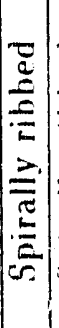 & 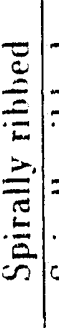 & 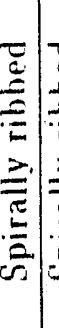 & 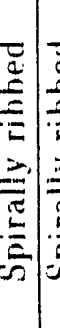 & 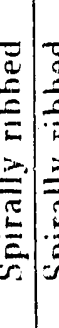 & 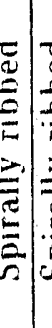 & 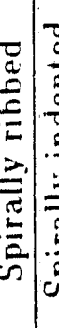 & 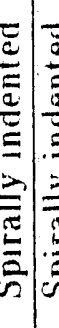 & 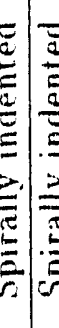 & 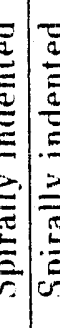 & 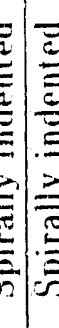 & 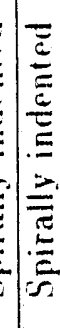 & 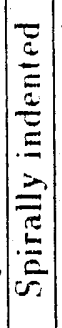 & 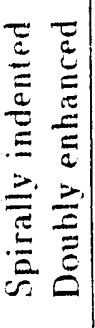 & 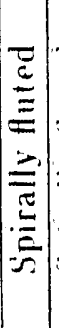 & \\
\hline$\frac{\sqrt[\pi]{\pi}}{\frac{a}{\pi}}$ & $\mid \begin{array}{l}5 \\
a \\
0 \\
0 \\
\vdots \\
\vdots\end{array}$ & 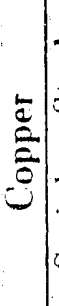 & 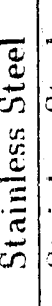 & 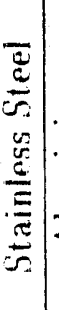 & 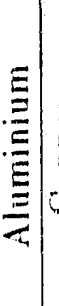 & & $\hat{\sigma}_{\bar{\sigma}}^{\circ}$ & ề & 岕 & ت. & 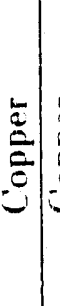 & לे & $\frac{5}{\hat{c}}$ & & $=$ & 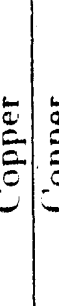 & & 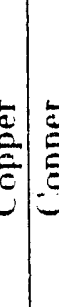 & $\underline{E}$ & 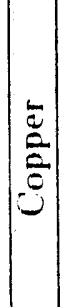 & $\varrho$ & 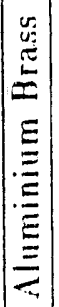 & \\
\hline$\frac{a}{2}$ & & 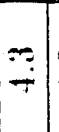 & 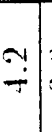 & $\because$ & $\underset{\sim}{2}$ & $\begin{array}{l}\bar{I} \\
=\underline{\Xi}\end{array}$ & 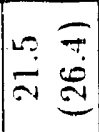 & 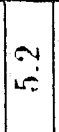 & $\stackrel{\varrho}{\Xi}$ & $=$ & $\because$ & $\infty$ & $\underset{x}{0}$ & $\stackrel{\square}{0}$ & 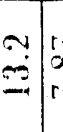 & $=$ & $\frac{7}{2}$ & 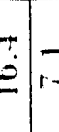 & 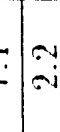 & 官 & $\begin{array}{l}4= \\
1=\end{array}$ & \begin{tabular}{l}
-1 \\
\hdashline-1 \\
\hdashline
\end{tabular} & $\stackrel{\alpha}{\circ}$ \\
\hline$\underline{-}$ & & $\stackrel{\vec{\perp}}{\bar{\Xi}}$ & 悉 & 点 & 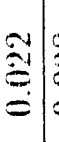 & 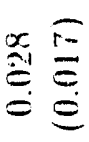 & 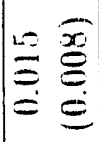 & $\stackrel{\Xi}{\Xi}$ & 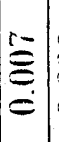 & 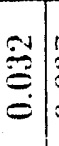 & 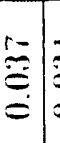 & 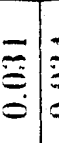 & 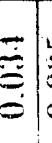 & $\stackrel{8}{=}$ & 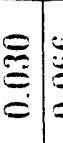 & $\stackrel{\mathscr{E}}{=}$ & $=$ & 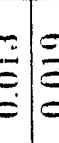 & 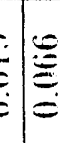 & 舀 & 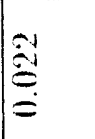 & 皇 & 尝 \\
\hline$=\frac{\tilde{T}}{\tilde{C}}$ & & $\stackrel{\square}{\doteqdot}$ & $\overrightarrow{-}$ & $\begin{array}{l}\vec{v} \\
\vec{\nabla}\end{array}$ & $\begin{array}{c}= \\
\dot{x} \\
s\end{array}$ & $\begin{array}{l}\overline{0} \\
\bar{g} \\
\bar{i}\end{array}$ & $\frac{\overline{\bar{p}}}{\bar{g}}$ & 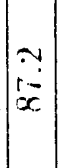 & $\left|\begin{array}{r}- \\
\dot{\alpha} \\
\dot{x}\end{array}\right|$ & 亲 & 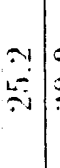 & $\begin{array}{l}\varrho \\
\vdots\end{array}$ & $\begin{array}{l}x \\
x\end{array}$ & 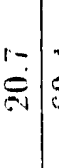 & $\vec{S}$ & $=$ & 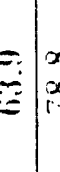 & \begin{tabular}{l|l}
$x$ \\
$x$ \\
$x$
\end{tabular} & $\frac{-1}{10}$ & 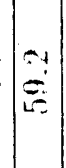 & $\because$ & 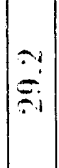 & $\bar{x}$ \\
\hline$=\bar{E}$ & & $=$ & $\stackrel{0}{0}$ & $\left|\begin{array}{l}\sim \\
i \\
i\end{array}\right|$ & 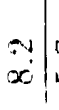 & 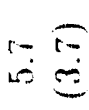 & 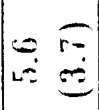 & $\therefore$ & $=$ & $=$ & $=$ & $\stackrel{x}{-}$ & 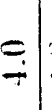 & $\underset{x}{x}$ & $=$ & & 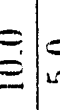 & $=$ & $=$ & $\begin{array}{l}\bar{\Xi} \\
\equiv\end{array}$ & $\begin{array}{l}\overline{1} \\
1-5\end{array}$ & $\mid$ & $\equiv$ \\
\hline$-\overline{\underline{\Xi}}$ & & $\overrightarrow{\tilde{x}}$ & $\Xi$ & 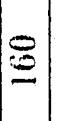 & $=$ & $\begin{array}{ll}2 & 0 \\
2 & 0 \\
\Xi & 0\end{array}$ & 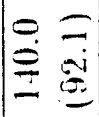 & 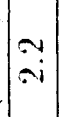 & $\underline{-}$ & 它 & 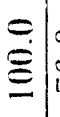 & $\dot{c}_{0}$ & 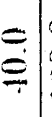 & 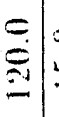 & $=$ & 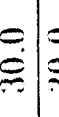 & 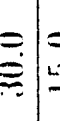 & $=$ & $=\alpha$ & $\begin{array}{l}\varrho \\
\stackrel{\Xi}{\Xi}\end{array}$ & $\bar{L}$ & 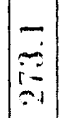 & 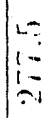 \\
\hline 12 & & $\stackrel{\sim}{\sim}$ & 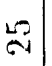 & $\vec{s}$ & $\varrho$ & $\approx 2$ & $\therefore 5$ & - & - & $=$ & $\stackrel{\sim}{\sim}$ & 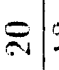 & 은 & $=$ & $=0$ & $=$ & $\because=$ & $\sigma r$ & $=0$ & $r$ & $1=$ & 7 & 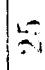 \\
\hline बE & & $\mid \begin{array}{c}2 \\
\tilde{\Xi} \\
0\end{array}$ & $=$ & $\left|\begin{array}{c}x \\
x \\
-2\end{array}\right|$ & 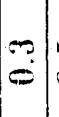 & 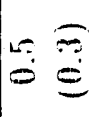 & $\stackrel{\bar{\Xi}}{\stackrel{\Xi}{=}}$ & $\frac{1}{0}$ & $\stackrel{\ominus}{\dot{\theta}}$ & $\stackrel{2}{=}$ & $\mid \begin{array}{l}0 \\
0 \\
=\end{array}$ & 0 & $\stackrel{5}{0}$ & $=$ & 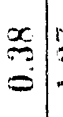 & $\ddot{\sim}$ & $\overline{0}: \overline{0}$ & & $=$ & $=$ & $\stackrel{21}{0}$ & $\begin{array}{l}0 \\
-i\end{array}$ & $\fallingdotseq$ \\
\hline$-\bar{E}$ & $\stackrel{-1}{=}$ & $\because$ & 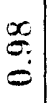 & $\ddot{z}$ & $=$ & $\vec{E}$ & $\stackrel{0}{0}=$ & $E$ & $\stackrel{\oplus}{=}$ & 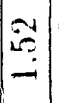 & $\underset{-}{\infty}$ & 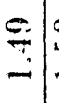 & 8 & - & $\stackrel{\infty}{=}$ & $\vec{\sim}$ & 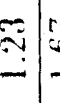 & $=$ & $E$ & $\stackrel{0}{-}$ & $\approx$ & $\stackrel{\sigma}{\sigma}$ & 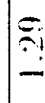 \\
\hline$-\bar{E}$ & 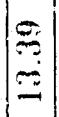 & $\frac{2}{2}$ & $\mid \begin{array}{c}\mathscr{E} \\
\tilde{\sigma} \\
\tilde{\sigma}\end{array}$ & $\mid$\begin{tabular}{l}
0 \\
0 \\
0 \\
\hdashline
\end{tabular} & $\mid \begin{array}{l}x \\
x \\
-2\end{array}$ & $\left\{\begin{array}{l}\infty \\
r-2 \\
-2\end{array}\right.$ & 10 & $\begin{array}{l}0 \\
\pm\end{array}$ & $\begin{array}{l}\equiv \\
\beth \\
\end{array}$ & $\left|\begin{array}{c}0 \\
\vdots \\
0 \\
0\end{array}\right|$ & $\mid$ & 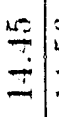 & $\begin{array}{l}E \\
\text { L } \\
-\end{array}$ & ב & 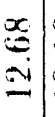 & 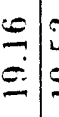 & $\begin{array}{l}2 \\
2 \\
2\end{array}$ & & 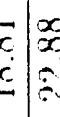 & \begin{tabular}{l}
$S$ \\
$\stackrel{S}{0}$ \\
\hdashline
\end{tabular} & $\frac{5}{3}$ & $\begin{array}{l}\infty \\
\infty \\
\infty\end{array}$ & 1 \\
\hline$\stackrel{\mathscr{g}}{ٍ ٍ}$ & $\begin{array}{c}0 \\
0 \\
\dot{y}\end{array}$ & $\sum_{5}$ & $\frac{\infty}{\frac{1}{1}}$ & $\stackrel{-\dot{\infty}}{\dot{S}}$ & $\dot{\bar{u}}$ & 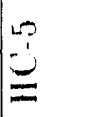 & 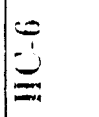 & $\stackrel{i}{z}$ & $\mid \begin{array}{l}\infty \\
\vdots \\
>\end{array}$ & $=$ & $\begin{array}{l}\varrho \\
\equiv\end{array}$ & $\bar{z}$ & $\frac{9}{7}$ & $\frac{1}{2}$ & $\frac{7}{1}$ & 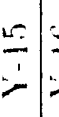 & $=$ & 12 & $\frac{0}{1}$ & $\approx$ & $\overline{-1}$ & $\therefore$ & \\
\hline
\end{tabular}




\begin{tabular}{|c|c|c|c|c|c|c|c|c|c|c|c|c|c|c|c|c|c|c|c|c|c|c|c|c|}
\hline$\frac{8}{2}$ & $\stackrel{\infty}{\infty}$ & $\mid \begin{array}{ll}0 \\
\dot{M}\end{array}$ & $=$ & 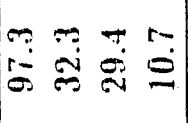 & $\mid$ & 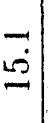 & -7 & : & 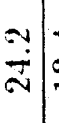 & $\overrightarrow{0}$ & 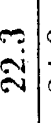 & 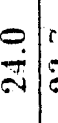 & 20 & $\begin{array}{l}0 \\
\dot{\vec{\omega}}\end{array}$ & $\overrightarrow{\vec{v}}$ & $\stackrel{i}{i}$ & $\underline{i}$ & $\ddot{0}$ & 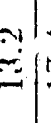 & $\div$ & & E & & $\begin{array}{l}2 \\
\alpha\end{array}$ \\
\hline 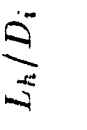 & $\underset{m}{\dddot{T}}$ & & & $\underset{\square}{\Xi}$ & $\mid \frac{\infty}{\infty}$ & $\begin{array}{l}\bar{\sigma} \\
\Xi\end{array}$ & 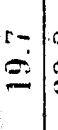 & $\stackrel{\infty}{\varrho}$ & 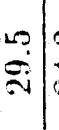 & $\frac{\sim}{\dot{\sim}}$ & $\overrightarrow{0}$ & $\begin{array}{l}3 \\
\stackrel{2}{2}\end{array}$ & $\begin{array}{l}\vec{a} \\
0 \\
0\end{array}$ & כִ & 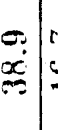 & 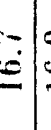 & $\stackrel{0}{=}$ & 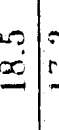 & 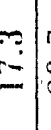 & $\ddot{2}$ & $\frac{x}{4}$ & 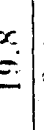 & & $\stackrel{\propto}{\varrho}$ \\
\hline$\frac{\ddot{6}}{5}$ & & & & 20 & $\underline{\square}$ & $\stackrel{r=}{\underline{\Xi}}$ & $\equiv$ & $\begin{array}{l}r \\
\infty\end{array}$ & $\Xi$ & $\stackrel{\bar{E}}{\stackrel{\Xi}{\Xi}}$ & $\stackrel{\square}{\Xi}$ & 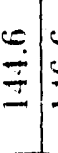 & $\stackrel{0}{=}$ & $\stackrel{0}{ \pm}$ & בְּ & & 我 & $\begin{array}{lll}0 & 0 \\
x & 0 & 0\end{array}$ & $\frac{x}{\infty}$ & $\underset{i=}{2}$ & & تئي & & $\stackrel{\circ}{\circ}$ \\
\hline$\stackrel{\square}{2}$ & $\overbrace{0}^{\infty}=$ & $\stackrel{-7}{=}$ & $\stackrel{\varrho}{\varrho}$ & $=\begin{array}{lll}a & 0 \\
\alpha & \alpha & \alpha \\
\alpha & \alpha\end{array}$ & & & & & & & & & & & & & & & & & & & & \\
\hline$\div \bar{E}$ & $\stackrel{\infty}{\equiv}$ & $\frac{T}{\dot{E}}$ & 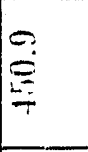 & 2 & $\bar{E}$ & $\frac{m}{\bar{x}}$ & $=$ & 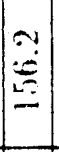 & $\begin{array}{l}0 \\
i \\
= \\
=\end{array}$ & $\begin{array}{l}c \\
\dot{a} \\
\dot{=}\end{array}$ & 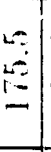 & $\begin{array}{l}0 \\
\dot{E} \\
\end{array}$ & 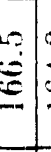 & $\stackrel{\bar{E}}{\dot{E}}$ & 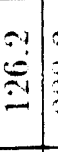 & $\begin{array}{l}x \\
x \\
x \\
x \\
x\end{array}$ & 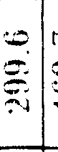 & 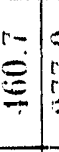 & 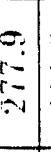 & 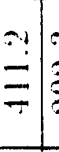 & 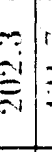 & $=$ & $\begin{array}{l}E \\
x \\
x \\
x\end{array}$ & $=$ \\
\hline$\div \frac{\pi}{E}$ & 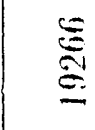 & & & $\equiv$ & 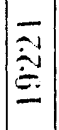 & 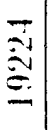 & $\overrightarrow{\widetilde{\vec{C}}}$ & 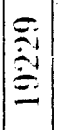 & $\begin{array}{l}\overrightarrow{1} \\
\vdots \\
=\end{array}$ & 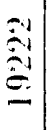 & 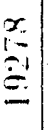 & 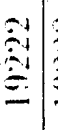 & 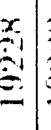 & | & 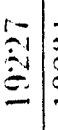 & 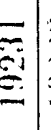 & 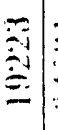 & $\equiv$ & $\vec{\vdots}$ & $\equiv$ & 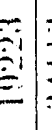 & 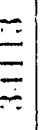 & 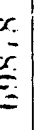 & 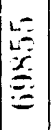 \\
\hline$\sqrt{\mathrm{E}}$ & $\bar{x}$ & 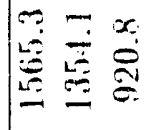 & $\mid \begin{array}{ll}0 \\
0\end{array}$ & 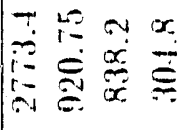 & 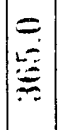 & 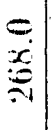 & 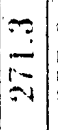 & 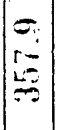 & $\mid \begin{array}{l}1- \\
\dot{x} \\
\dot{C}\end{array}$ & $\stackrel{=}{x}$ & 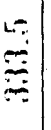 & 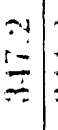 & & 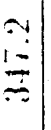 & 㐌 & $\stackrel{\square}{-1}$ & 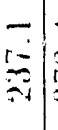 & 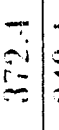 & $\begin{array}{l}\bar{\vdots} \\
\overline{\dot{\Delta}}\end{array}$ & 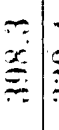 & 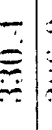 & 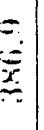 & $\begin{array}{l}= \\
\frac{x}{2}\end{array}$ & 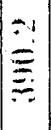 \\
\hline$\leq \widehat{\Xi}$ & $\begin{array}{l}\sigma \\
\alpha \\
\alpha\end{array}$ & & & $\frac{0}{a x}$ & $\bar{\Xi}$ & 产 & $\begin{array}{l}= \\
\therefore= \\
\therefore=\end{array}$ & $\bar{\vdots}$ & $\begin{array}{l}-\bar{\square} \\
\bar{\varphi}\end{array}$ & $\frac{\alpha}{\alpha}$ & 品 & $\bar{\square}$ & $\stackrel{\square}{\stackrel{\Xi}{\Xi}}$ & דיב- & $\begin{array}{c}= \\
z\end{array}$ & $\bar{\vdots}$ & 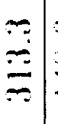 & $=$ & \begin{tabular}{l}
$\bar{Z}$ \\
\hdashline \\
\hdashline
\end{tabular} & $\bar{Z}$ & $\frac{-1}{-3}$ & $\bar{\Xi}$ & $=$ & $\mid$\begin{tabular}{l}
0 \\
$\vdots$ \\
\hdashline
\end{tabular} \\
\hline : & & & & $\bar{\alpha} \bar{\alpha}$ & 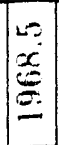 & 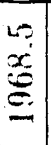 & 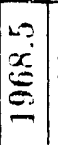 & 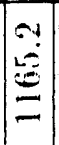 & 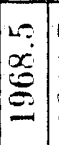 & $\begin{array}{l}\bar{Z} \\
\bar{z}\end{array}$ & $\begin{array}{l}0 \\
0 \\
0 \\
5 \\
2\end{array}$ & 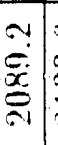 & $\frac{0}{2}$ & $\begin{array}{l}\infty \\
\stackrel{\infty}{2} \\
2\end{array}$ & 污 & $\bar{\Xi}$ & $\vec{E}$ & $=$ & $\bar{E}$ & $=$ & & 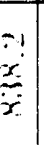 & 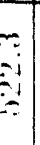 & 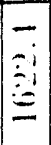 \\
\hline$\because \bar{\Xi}$ & ${ }_{\vec{N}} \Theta$ & $\bar{\sigma}$ & $\vec{v}$ & $\vec{L} \stackrel{\mathscr{\alpha}}{\varrho} \stackrel{\vec{\alpha}}{\varrho}$ & & & & & & & & & & & & & & & & & & & & \\
\hline$\theta-\bar{E}$ & 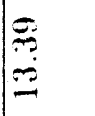 & $\frac{2}{9}$ & 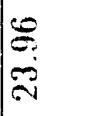 & 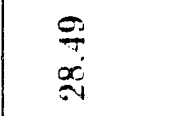 & $\begin{array}{l}\dot{\infty} \\
\ddot{c}\end{array}$ & $\begin{array}{l}x \\
\therefore \\
\therefore\end{array}$ & $\begin{array}{l}\overrightarrow{0} \\
-\end{array}$ & $\begin{array}{l}\varrho \\
\underline{\Xi}\end{array}$ & $\begin{array}{c}\mathscr{P} \\
\dot{Z}\end{array}$ & $\begin{array}{l}\nexists \\
0 \\
2\end{array}$ & $\begin{array}{l}2 \\
= \\
=\end{array}$ & $=$ & 告 & 呈 & 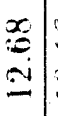 & 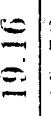 & $\begin{array}{l}2 \\
= \\
=\end{array}$ & 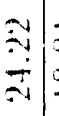 & $\begin{array}{l}\bar{x} \\
\alpha \\
\bar{x}\end{array}$ & $\begin{array}{l}\infty \\
\alpha \\
\dot{\alpha} \\
\dot{\sigma}\end{array}$ & $\begin{array}{l}\Xi \\
\vdots \\
\vdots\end{array}$ & 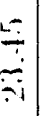 & 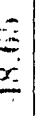 & \begin{tabular}{l}
$1=$ \\
$1 \div$ \\
\hdashline
\end{tabular} \\
\hline$\stackrel{\mathscr{E}}{\Xi}$ & în & $\underset{\xi}{\xi}$ & $\sum_{\infty}^{\infty}$ & $\sum_{0}^{P}$ & 勇 & $\begin{array}{l}0 \\
\vdots \\
\vdots \\
\end{array}$ & $\mid \begin{array}{l}0 \\
\vdots \\
\vdots\end{array}$ & \begin{tabular}{l}
$1-$ \\
\hdashline \\
$z$
\end{tabular} & $\begin{array}{l}x \\
\vdots \\
z\end{array}$ & 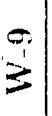 & $\begin{array}{l}0 \\
\frac{1}{3}\end{array}$ & $\bar{z}$ & $\frac{2}{3}$ & $\frac{2}{3}$ & \pm & $=$ & $\begin{array}{c}\epsilon \\
-1\end{array}$ & $\bar{z}$ & $\frac{x}{2}$ & $\frac{1}{2}$ & 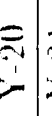 & $\vec{\nabla}$ & & $\because$ \\
\hline
\end{tabular}


$A_{h} \quad=$ heated area, $\pi D_{i} L_{h}, \mathrm{~mm}^{2}$

$A_{x}=$ cross-sectional area, $\pi D_{i}^{2} / 4, \mathrm{~mm}^{2}$

$\alpha \quad=$ belix angle, degrees

$D_{1} \quad=$ maximum internal diameter, $\mathrm{mm}$

$\epsilon=$ roughness beight, $\mathrm{mm}$

$e / D_{i} \quad=$ relative roughness beight

$L_{e} \quad=$ smooth entrance length, $\mathrm{mm}$

$L_{e, r} \quad=$ rough entrance length. $\mathrm{mm}$

$L_{h} \quad=$ heated length, $\mathrm{mm}$

$L_{p} \quad=$ distance between pressure taps, $\mathrm{mm}$

$L_{e} / D_{i}=$ relative smooth entrance length

$L_{e, r} / D_{i}=$ relative rough entrance length

$L_{h} / D_{i}=$ relative beated length

$L_{p} / D_{1}=$ relative pressure drop measurement length

$l=\operatorname{lead}, l=\pi D_{1} / \tan \alpha=p N_{s}, \mathrm{~mm}$

$N_{s} \quad=$ number of starts

$p \quad=$ axial pitch, $\mathrm{mm}$

p/e $\quad=$ relative pitch

$t \quad=$ wall thickness 


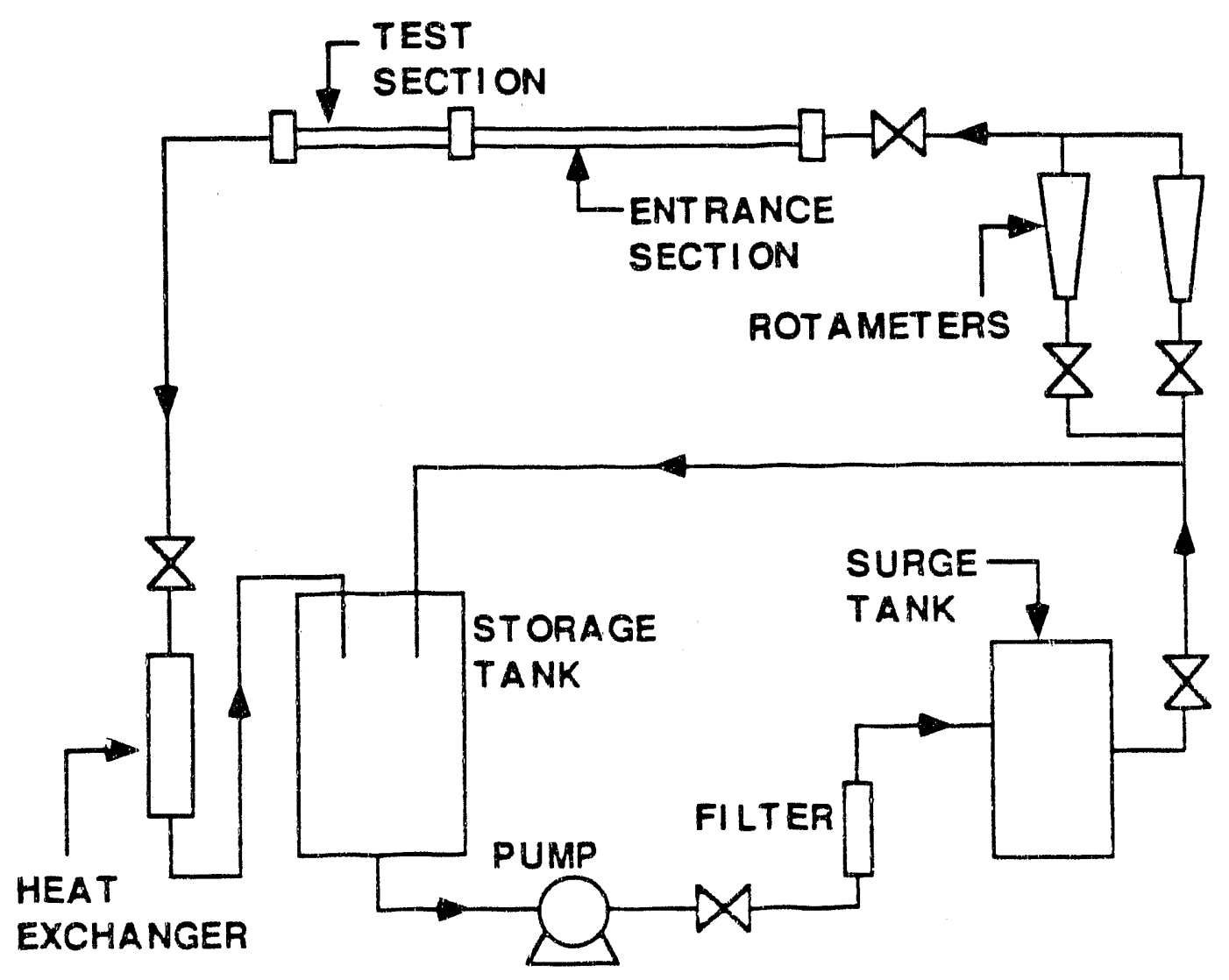

Figure 3. Schematic of liquid test facility. 


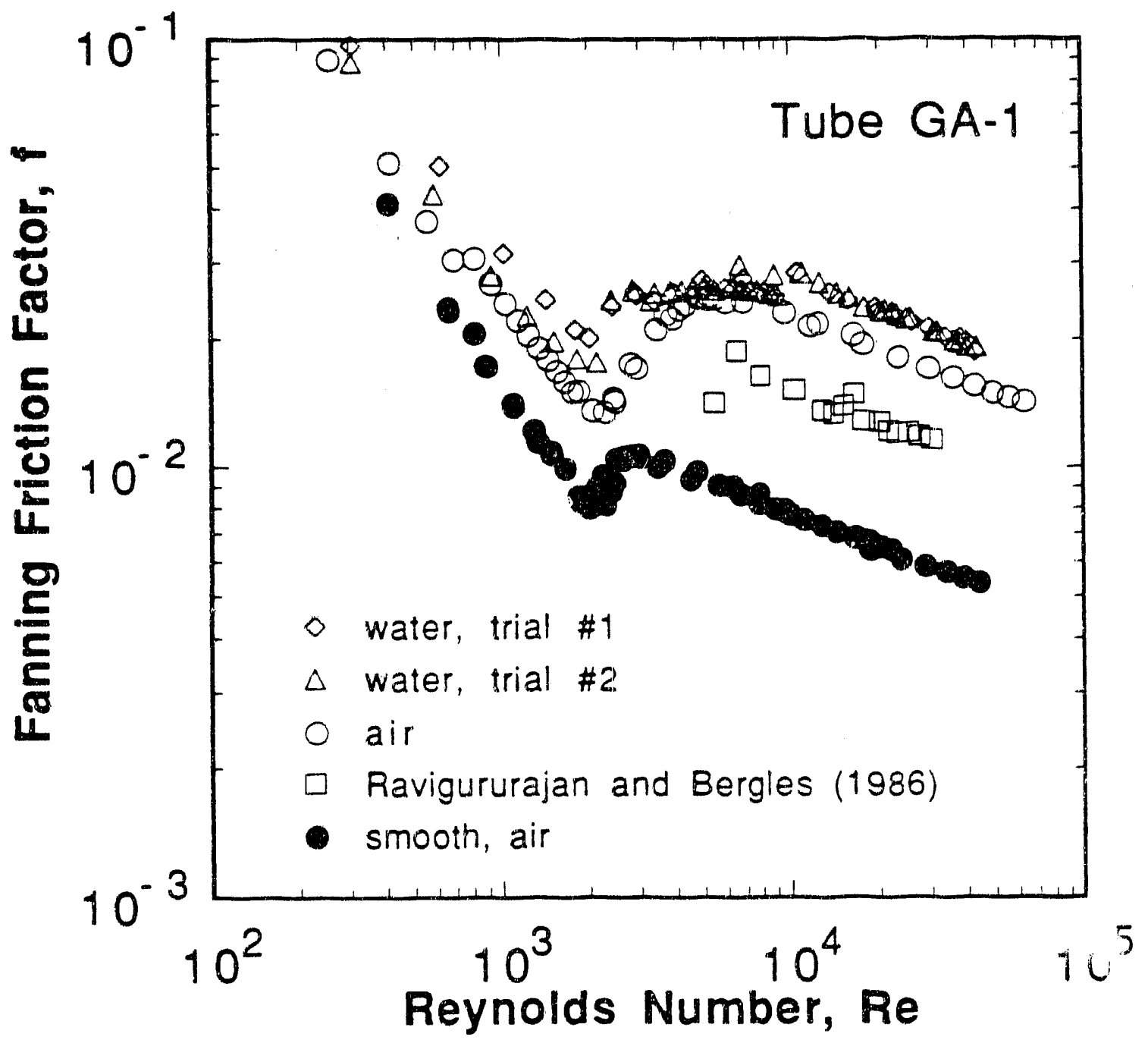

Figure 1. Comparison of air and water data for GA-1. 


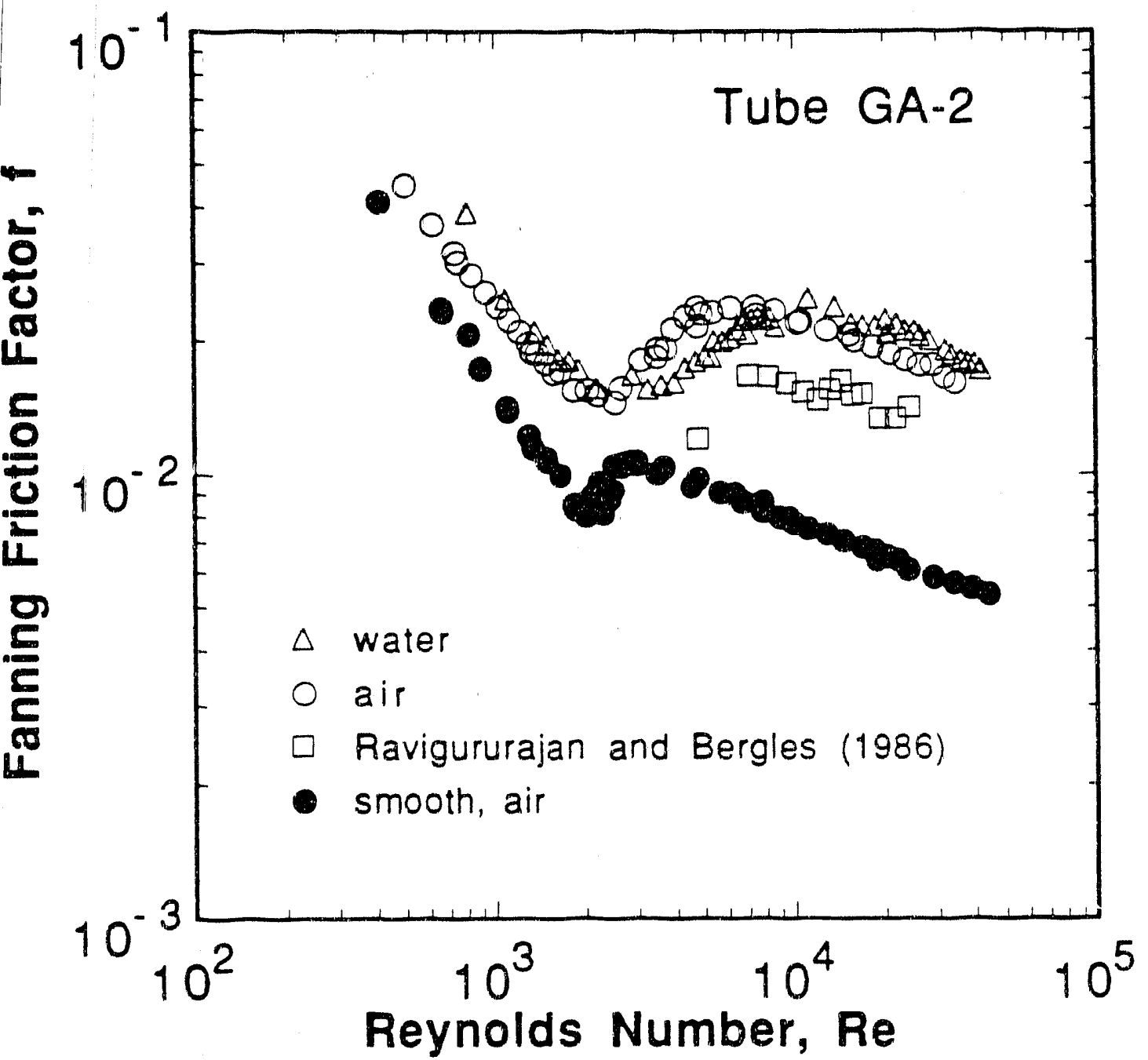

Figure 2. Comparison of air and water data for GA-2 


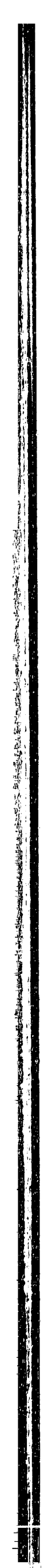

